\title{
Two Interdependent TRPV Channel Subunits, Inactive and Nanchung, Mediate Hearing in Drosophila
}

\author{
Zhefeng Gong, ${ }^{1 \star}$ Wonseok Son, ${ }^{2 \star}$ Yun Doo Chung, ${ }^{3 \star}$ Janghwan Kim,,${ }^{2,6}$ Dong Wook Shin, ${ }^{2}$ Colleen A. McClung, ${ }^{1}$ \\ Yong Lee, ${ }^{4}$ Hye Won Lee, ${ }^{2}$ Deok-Jin Chang, ${ }^{4}$ Bong-Kiun Kaang, ${ }^{4}$ Hawon Cho, ${ }^{5}$ Uhtaek Oh, ${ }^{5}$ Jay Hirsh, ${ }^{1}$ \\ Maurice J. Kernan, ${ }^{3}$ and Changsoo $\mathrm{Kim}^{2,7}$ \\ ${ }^{1}$ Biology Department, University of Virginia, Charlottesville, Virginia 22904, ${ }^{2}$ Department of Genetics, Hanwha Chemical Company R\&D Center, Sinsung- \\ Dong, Yusung-Gu, Daejeon 305-345, Korea, ${ }^{3}$ Department of Neurobiology and Behavior, State University of New York at Stony Brook, New York 11794, \\ ${ }^{4}$ National Research Laboratory, School of Biological Sciences, Seoul National University, Seoul 151-742, Korea, ${ }^{5}$ Sensory Research Center, Creative Research \\ Initiatives, Seoul National University, Seoul 151-742, Korea, ${ }^{6}$ Department of Microbiology, Chungnam National University, Daejeon 305-764, Korea, and \\ ${ }^{7}$ School of Biological Sciences and Technology, Chonnam National University, Gwangju 500-757, Korea
}

Hearing in Drosophila depends on the transduction of antennal vibration into receptor potentials by ciliated sensory neurons in Johnston's organ, the antennal chordotonal organ. We previously found that a Drosophila protein in the vanilloid receptor subfamily (TRPV) channel subunit, Nanchung (NAN), is localized to the chordotonal cilia and required to generate sound-evoked potentials (Kim et al., 2003). Here, we show that the only other Drosophila TRPV protein is mutated in the behavioral mutant inactive (iav). The IAV protein forms a hypotonically activated channel when expressed in cultured cells; in flies, it is specifically expressed in the chordotonal neurons, localized to their cilia and required for hearing. IAV and NAN are each undetectable in cilia of mutants lacking the other protein, indicating that they both contribute to a heteromultimeric transduction channel in vivo. A functional green fluorescence protein-IAV fusion protein shows that the channel is restricted to the proximal cilium, constraining models for channel activation.

Key words: channel; deafness; Drosophila; hair cell; hearing; mechanosensory

\section{Introduction}

The TRPV family comprises a subset of the TRP superfamily of cation channel subunits (Caterina et al., 1997, 1999, 2000; Colbert et al., 1997; Caterina and Julius, 2001). Different TRPV channels are activated by physical stimuli, including heat and osmotic swelling, as well as ligands, including protons, vanilloids, and phorbol esters (Caterina et al., 2000; Benham et al., 2003; Gao et al., 2003; Vriens et al., 2004; for review, see Nilius et al., 2004).

The TRPV gene family has diverged in vertebrates and invertebrates from a single ancestral gene. Two main invertebrate branches are represented in the nematode Caenorhabditis by osm-9 and by four ocr genes, respectively (Colbert et al., 1997; Tobin et al., 2002). When coexpressed in ciliated sensory neurons, OSM-9 and OCR-2 localize to sensory cilia to transduce

\footnotetext{
Received April 29, 2004; revised Aug. 4, 2004; accepted Aug. 4, 2004.

This work was supported by National Research Laboratory Grant M1-0104-00-0140 (B.-K.K.), Creative Research Initiatives of the Ministry of Science and Technology of Korea and, in part, by a BK21 program (U.0.) and National Institutes of Health Grants DC02780 (M.J.K.) and GM27318 (J.H.). We thank Seymour Benzer for the mAb 22C10 and the anonymous reviewers whose comments greatly improved this manuscript.

${ }^{*}$ Z.G., W.S., and Y.D.C. contributed equally to this work.

Correspondence should be addressed to either of the following: Jay Hirsh at the above address, E-mail: jh6u@virginia.edu, or Changsoo Kim, School of Biological Sciences and Technology, Chonnam National University, Gwangju 500-757, Korea, E-mail: changgk2001@hanmail.net.

Y. D. Chung's present address: Department of Life Science, University of Seoul 130-743, Korea.

C. A. McClung's present address: Department of Psychiatry, University of Texas Southwestern Medical Center, Dallas, TX 75390.

DOI:10.1523/JNEUROSCI.1645-04.2004

Copyright $\odot 2004$ Society for Neuroscience $\quad 0270-6474 / 04 / 249059-08 \$ 15.00 / 0$
}

aversive hyperosmotic and mechanosensory stimuli. The Drosophila genome includes one representative of each of the two invertebrate branches. Nanchung (NAN), related to the OCR proteins, is activated by hypotonic swelling in cultured cells and required to transduce near-field sound (Kim et al., 2003).

Hearing requires a mechanically activated channel to transduce sound-induced displacement into neuronal membrane depolarization (Strassmaier and Gillespie, 2002). In mammals, this occurs in the cochlear hair cells. The hair cell transducer channel properties resemble those of TRP superfamily channels, but its identity is unknown (Gillespie and Walker, 2001; Strassmaier and Gillespie, 2002). TRPV4 is expressed in the mammalian inner ear (Liedtke et al., 2000), but mice lacking TRPV4 have normal hearing (Liedtke and Friedman, 2003). Another candidate is the mechanosensory channel NOMPC, which is functionally required in zebrafish hair cells (Sidi et al., 2003). Surprisingly, no NOMPC homologs appear in mammalian genomes (Corey, 2003; Sidi et al., 2003).

Hearing in Drosophila depends on antennal movement by bulk air displacement, close to sound sources such as the vibrating wing of a courting male fly (Eberl, 1999). Vibration of distal antennal segments is transmitted by extracellular dendritic caps to sensory cilia in the antennal chordotonal organ (Eberl et al., 2000). The NAN protein is localized to the chordotonal cilia and is required for auditory transduction (Kim et al., 2003). The interaction of OCR-2 with OSM-9 in C. elegans suggested that NAN might interact with a partner TRPV subunit. 
We found that the remaining Drosophila TRPV protein is encoded by inactive (iav), a locus previously associated with a variety of mutant phenotypes: motor inactivity, courtship abnormalities, low levels of the neurotransmitters tyramine and octopamine, and aberrant behavioral responses to cocaine (Homyk and Sheppard, 1977; Kaplan, 1977; O’Dell et al., 1987; O'Dell, 1993, 1994; McClung and Hirsh, 1999). We show that iav mutants are deaf and that IAV and NAN require each other for expression in cilia. Interestingly, a functional green fluorescent protein (GFP)-tagged IAV protein is restricted to the proximal part of the cilia. We propose that IAV-NAN channels are gated by forces transmitted through the ciliary membrane or cytoskeleton rather than by direct interaction with the dendritic cap.

\section{Materials and Methods}

Drosophila stocks and growth conditions. The iav ${ }^{1}$ line was obtained from Paul Salvaterra (Beckman Institute, Duarte, CA). All of the deficiency and P-element lines, except the deletions of the P insertion EP1613, came from the Bloomington (IN) and Umea (Sweden) Drosophila stock centers. EP1613 deletions were kindly provided by Ulrike Gaul (Rockefeller University, New York, NY). Flies were grown on standard media containing cornmeal, yeast, and agar.

Isolation of $\operatorname{iav}^{3621}$. Male flies were mutagenized with ethyl methanesulfonate (Lewis and Bacher, 1968) and crossed to $\widehat{\mathrm{XXY}}$ females. Male progeny carrying the mutagenized $\mathrm{X}$ chromosomes were screened for a phenotype observed in $i a v^{l}$, slow recovery from $\mathrm{CO}_{2}$ anesthesia. Groups of 30 flies were assessed for wall climbing ability after startle. Climbingcapable flies were $\mathrm{CO}_{2}$ anesthetized for $30 \mathrm{sec}$, and those unable to climb the wall after 5 min were collected and checked by crossing to $i a v^{1}$ and repeating the screening procedure. One valid noncomplementing iav mutant, $i a v^{3621}$, was found after screening 5000 mutagenized X chromosomes. This allele behaves like $i a v^{l}$ in recovery from $\mathrm{CO}_{2}$ anesthesia, and female $i a v^{1} / i a v^{3621}$ heterozygotes are slow to recover from $\mathrm{CO}_{2}$ anesthesia as well.

Polymorphic markers and mapping crosses. Four length polymorphisms were isolated in the iav candidate region by screening $w^{1118}$ iav $^{1}$ and $P\left[w^{+}\right] w^{1118}$ lines with PCR primers flanking short low-complexity sequences. The primers used to detect these length polymorphisms are shown in supplemental materials.

Mapping crosses were set up to allow recombination between $E P\left[w^{+}\right]$ $\mathrm{P}$ elements in the iav candidate region and the $w^{1118} \mathrm{iav}^{1}$ chromosome, scoring for phenotypically inactive $w^{+}$flies. Recombination was done in the presence of a $\mathrm{P}$ element jumpstarter chromosome in an attempt to generate recombinants at the P element sites (Preston and Engels, 1996), but based on analyses of the flanking markers, no such events were observed, and no cases of $\mathrm{P}$ elements hopping to homologous chromosomes were observed. The insertions site $\mathrm{P}$ element $\mathrm{P} 11855$ responsible for l(1)G0254 was recovered by inverse PCR (Rehm, 2003) and found to lie within the predicted gene CG4094.

Cloning of iav $c D N A$. The transcription start and termination sites were determined by FirstChoice RLM-rapid amplification of cloned ends (RACE) (Ambion, Austin, TX). iav cDNA was prepared from D. melanogaster (Canton-S) poly-A tailed RNA using Superscript II (Invitrogen, San Diego, CA) and amplified by the PCR amplification (Turbo Pfu; Stratagene, La Jolla, CA). The N-terminal region was obtained using the following primers: 5'-CGAGCTCGTTGAGTTCGGAGCAGGGATAGAAATC-3', 5'-AGGTCTTCCACTTCTCCTCCAGCAGG-3'. The C terminus was obtained using the following primers: $5^{\prime}$-CGGAGGTCTTTCGTGAAATGTTGGAG-3', 5' -ACATCAACAAGATCCTGGCCAGAAAGTGGGTACC-3'. Each PCR fragment was cloned into pT-Adv (Clontech, Cambridge, UK), and both strands were sequenced. The two fragments were joined using EcoRI to make full-length iav cDNA.

Transformation rescue. Germline transformation was essentially the same as that described previously (Spradling and Rubin, 1982). The DNA segment used extended from an $\mathrm{XbaI}$ site $2.4 \mathrm{~kb}$ upstream to a site $3 \mathrm{~kb}$ downstream of the predicted CG4536 transcription unit and was incorporated into the $\mathrm{XbaI}$ site of pCaspeR4. Germline transformation was performed by injecting this recombinant plasmid and "wings clipped" helper into $w$ iav ${ }^{1}$ embryos. GFP-IAV fusions were constructed by inserting the GFP coding sequence either at an SmaI site in exon 1 or at a HindIII site in exon 7. The genomic segment of iav used in these constructs extended from an NruI site in the upstream gene CG4532 to a NotI site downstream from iav (supplemental material, available at www.jneurosci.org).

In situ hybridization. The iav antisense and sense RNA were prepared from 3' $2.5 \mathrm{~kb}$ of iav cDNA by using digoxigenin (DIG) RNA labeling kit (Roche Products, Hertforshire, UK). Wild-type embryos were collected after $16 \mathrm{hr}$ egg laying and stained with DIG-labeled iav RNA probes as described by Skeath and Carroll (1994). Samples were mounted in $90 \%$ glycerol and observed with a Zeiss Axioplan 2 microscope (Zeiss, Thornwood, NY).

Production of anti-IAV sera and immunohistochemistry. Fragments encoding the $\mathrm{N}$ terminus (amino acids 1-130) and the $\mathrm{C}$ terminus (amino acids 956-1123) of IAV were subcloned into pGEX4T (Amersham Biosciences, Arlington Heights, IL) to create pGST-IavN and pGST-IavC, respectively. The glutathione $S$-transferase (GST) fusion proteins were expressed in Escherichia coli (BL21), purified by the gel extraction or by the use of GST-affinity column, and subsequently used to immunize rats. Adult heads were horizontally sectioned and immunostained as described previously (Wolff, 2000). Anti-IAV sera were used at 1:500 to 1:1000 dilution, and the monoclonal antibody (mAb) 22C10 (a gift from Seymour Benzer, Caltech, Pasadena, CA) was used at 1:500 dilution. Secondary antibodies were FITC-conjugated anti-rat goat IgG (1:100 dilution; Jackson ImmunoResearch, West Grove, PA) and cyanine 3 (Cy3)-conjugated anti-mouse goat IgG (1:500 dilution; Jackson ImmunoResearch). Stained samples were observed with a confocal microscope (Zeiss with Bio-Rad laser system; Bio-Rad, Hercules, CA)

Measurement of intracellular $\mathrm{Ca}^{2+}$. Chinese hamster ovary (CHO) K1 cells were transfected using Lipofectamine 2000 (Invitrogen) with the iav cDNA expression vector in which an iav cDNA fragment cleaved by NotI and $K p n I$ was cloned into pcDNA3.1mychis $(-) B$. Stable cell lines were selected in G418-containing $(100 \mu \mathrm{g} / \mathrm{ml})$ culture media (RPMI 1640 with $10 \%$ fetal bovine serum, $100 \mu \mathrm{g} / \mathrm{ml}$ penicillin $\mathrm{G}$, and $0.0085 \%$ streptomycin).

Stably transfected cells were cultured on cover glasses, attached to an imaging chamber, and $5 \mu \mathrm{M}$ fluo4/AM (Molecular Probes, Eugene, OR) was loaded for $60 \mathrm{~min}$ at $37^{\circ} \mathrm{C}$ in iso-osmotic saline solution (294 mOsm/ $\mathrm{kg}$ ) containing the following (in $\mathrm{mM}$ ): $130 \mathrm{NaCl}, 2.5 \mathrm{KCl}, 2 \mathrm{CaCl}_{2}, 1$ $\mathrm{MgCl}_{2}, 20$ D-glucose, and 10 HEPES, pH 7.3 (Liedtke et al., 2000). The fluorescence signal was visualized by confocal microscopy (LSM 510; Zeiss). Analysis of single-cell signal intensity, referred to arbitrary unit in Figure 3, was performed on Window NT running NIH Image software. Solutions with different osmotic strengths were made by adjusting mannitol concentration. The test solutions included a grade series of hypotonic solutions $(219,239$, and $264 \mathrm{mOsm} / \mathrm{kg}$ ), a hypertonic solution (330 $\mathrm{mOsm} / \mathrm{kg}$ ), and isotonic solutions (294 mOsm $/ \mathrm{kg}$ ). The osmolality of all solutions was checked with freezing point checking osmolality meter (Knauer, Berlin, Germany).

Patch-clamp recordings. The iav expression vector was transiently transfected into human embryonic kidney (HEK) 293T cells, and the transfected cells were used for recording. Whole currents were recorded with an Axopatch 200B amplifier (Axon Instruments, Foster City, CA). Patch recordings were performed at room temperature $24-48 \mathrm{hr}$ after transfection. Borosilicate glass patch pipettes had resistances of 3-5 $\mathrm{MOhm}$ when filled with the isotonic intracellular solution. To measure channel currents, cells were held at a potential of $0 \mathrm{mV}$ and subjected to $1 \mathrm{sec}$ voltage ramps from -100 to $100 \mathrm{mV}$. Signals were filtered at $1 \mathrm{kHz}$ using a four-pole low-pass Bessel filter digitized at 200 samples/msec using Digidata 1200 (Axon Instruments) and stored in a computer. pClamp8 software (Axon Instruments) was used to control the amplifier and to acquire the data. Pipette and bath solutions contained $5 \mathrm{~mm}$ HEPES, 1 mm EDTA, $120 \mathrm{~mm} \mathrm{NaCl}$, and $100 \mathrm{~mm}$ Mannitol (321 mOsm/ $\mathrm{kg}$ ). To activate the currents, cells were superfused while recording with a hypotonic solution containing only 5 mM HEPES, 1 mM EDTA, and 120 $\mathrm{mm} \mathrm{NaCl}(221 \mathrm{mOsm} / \mathrm{kg})$. In bi-ionic conditions, $120 \mathrm{~mm} \mathrm{KCl}$ was substituted for $\mathrm{NaCl}$ in the bath solutions. 


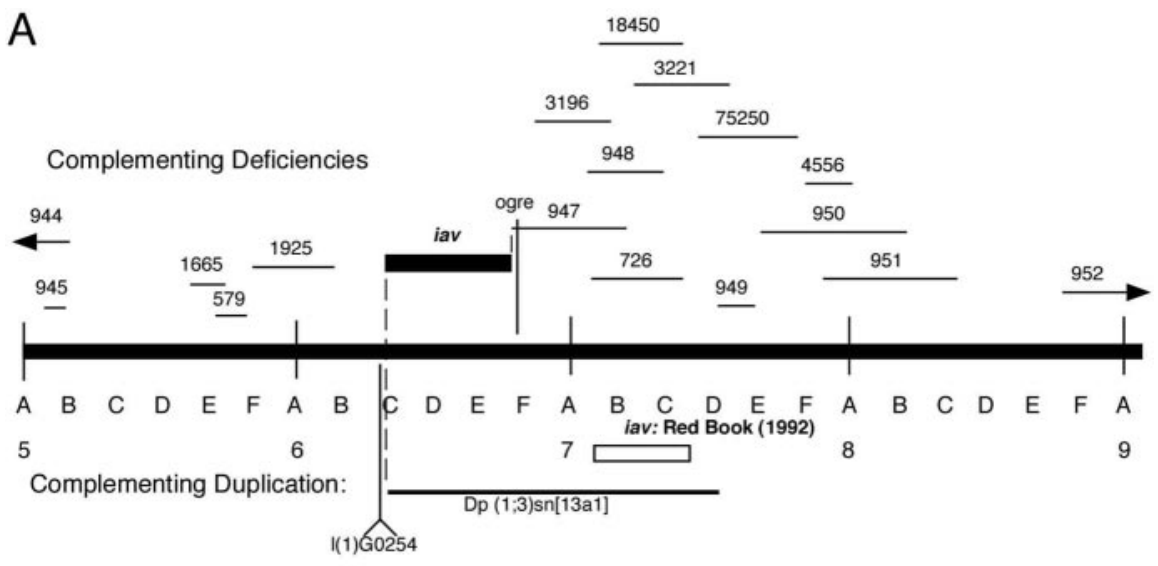

B

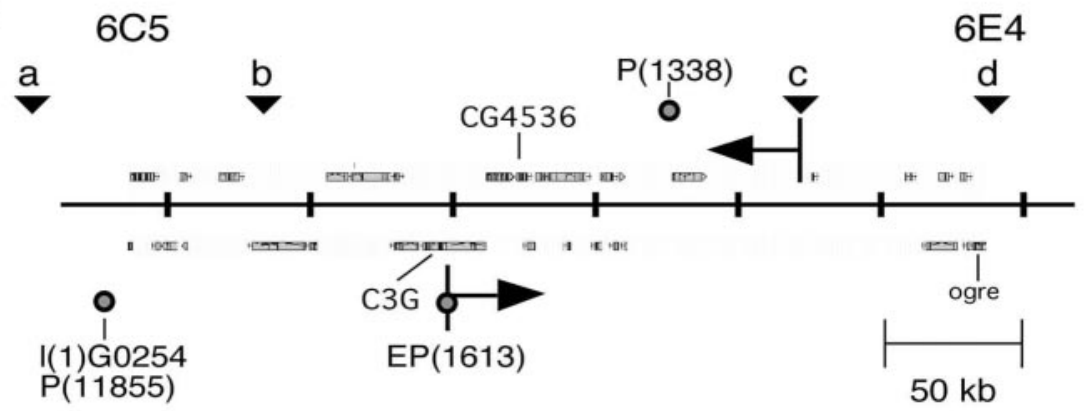

C

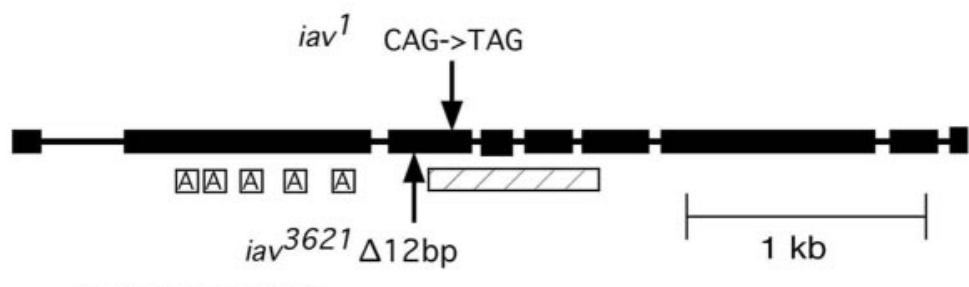

ه ANKYRIN REPEAT

TRANSMEMBRANE DOMAIN

Figure 1. Mapping the iav locus. A, Deficiency-duplication mapping. The indicated deficiencies and duplication were crossed to iav $^{7}$ and scored for ability to complement the locomotor inactivity phenotype. The right edge of the candidate region is defined by the gene ogre, which complements iav and is to the left of $D f(1) 3196$ but is uncovered by $D f(1) 947$ (Curtin et al., 1999). The left edge is defined by the lethal P element insertion I(1)G0254 (Schaefer et al., 1999), which is in the predicted gene (G4094 in 6C10-11 (data not shown). This insertion is not rescued by $D p(1 ; 3) s n[s n 13 a 1]$, indicating that it is left relative to the duplication. $B$, The iav candidate region at higher resolution assembled from Flybase (The FlyBase Consortium, 2003). Predicted genes are shown above and below the line representing the genetic region. The circles show P-element insertions, and inverted triangles $a-d$ represent sequence polymorphisms used for recombination mapping (see Materials and Methods). The left extent of the candidate region is defined by the lethal $P$ insertion I(1) G0254 in cytogenetic region $6 \mathrm{C} 5$ and the right by the gene ogre in $6 \mathrm{E} 4$, because Df (1)3196 (Df(1)SXI-bt) removes ogre yet complements iav (Curtin et al., 1999). C, (G4536 gene structure and lesions in the iav alleles. Predicted structure of the iav protein and the iav mutant alleles. Gene structure prediction is from Flybase release 3.1 (The FlyBase Consortium, 2003) and from PCR 5' and 3' RACE analyses and sequencing.

Johnston's organ electrophysiology. Sound-evoked potentials were recorded from Johnston's organ as described previously (Eberl et al., 1997, 2000). Briefly, mutant or control flies were anesthetized by chilling and then immobilized in the end of a cut-out plastic micropipet tip with the head exposed and the antennae free to vibrate. Electrolytically sharpened tungsten electrodes were inserted into an antenna between the first and second segments and into the head. The electrodes were connected to a DAM-50 differential amplifier (World Precision Instruments, Sarasota, $\mathrm{FL}$ ), and the amplified differential AC voltage was acquired and digitized using an Instrunet analog-to-digital converter and Superscope II software (GW Instruments, Somerville, MA). Sound stimuli generated with Superscope were sent via Instrunet to a Realistic MPA-30 audio amplifier (Radio Shack, Fort Worth, TX) and delivered to the fly via 0.25 inch (inner diameter) Tygon tubing. To ensure near-field acoustic conditions in which the particle velocity component of sound predominates, flies were mounted so that antennae were within one radius of the center of the tube opening. Typically, the responses to 10 trains of five sound pulses were averaged. Recordings were made alternately from control and mutant flies.

\section{Results \\ The inactive locus encodes a TRPV channel subunit}

To map iav, we tested a series of $\mathrm{X}$ chromosome deletions for complementation of the locomotor phenotype (inactivity) of $i a v^{1}$ mutants. All deletions tested complemented iav mutant inactivity, including deletions of the region 7A5-C1, the location of iav previously reported in the Drosophila"Red Book" (Hall, 1992). A segment of chromosome region 6 is not uncovered by any available deletion. The duplication $D p(1 ; 3) s n^{13 a 1}$, which covers part of this gap, complemented the iav inactivity, placing the gene within the cytogenetic interval 6C5-E4 (Fig. 1A). The distal molecular limit of this interval is the lethal $\mathrm{P}$ insertion l(1)G0254 in the predicted gene CG4094; the proximal limit is the ogre gene. The interval contains $\sim 300 \mathrm{~kb}$ of DNA with 30 predicted transcription units (The FlyBase Consortium, 2003) and includes the sites of several $\mathrm{P}$ element insertions, each of which complemented iav (data not shown). To subdivide the region, we mapped four polymorphisms (Fig. $1 B$, $a-d$ ) (supplemental material, available at www.jneurosci.org) between a $w^{1118}$ iav $^{1}$ line and $w^{1118}$ lines containing $P\left[w^{+}\right]$insertions in the iav candidate regions. Analysis of these polymorphisms in $w^{+}$iav $v^{1}$ recombinants between $w^{1118} i a v^{1}$ and $w^{1118}$ $P\left[w^{+}\right]$chromosomes indicated that iav was located between the $\mathrm{P}$ insertion $P[E P] C 3 G^{E P 1613}$ and polymorphism c.

The reduced interval contains $20 \mathrm{~kb}$ of coding DNA in 14 predicted genes. We sequenced protein-coding exons and adjoining small introns from within this region and found significant changes in only one predicted gene, CG4536. This gene encodes a TRPV family ion channel with five predicted ankyrin repeats in the cytoplasmic amino terminal and an ion channel domain containing six transmembrane domains (Fig. 1C). We obtained the full-length cDNA from CG4536 by reverse transcription (RT)-PCR after $5^{\prime}$ and $3^{\prime}$-ends of the CG4536 transcript were determined by RACE. The 1123-amino acid open reading frame is in complete agreement with the CG4536 gene prediction except for a single amino acid polymorphism $448 \mathrm{G} \rightarrow$ A. As described previously (Liedtke et al., 2000; Kim et al., 2003), the iav gene product is most similar in sequence to Caenorhabditis OSM-9 (43\% amino acid identity), with decreasing similarity to the Drosophila Nanchung and Caenorhabditis OCR proteins (29\% amino acid identity), and the mammalian TRPV channels (19\% average 
amino acid identity) (supplemental material, available at www.jneurosci.org).

The lesion in $i a v^{1}$, C1363T in the third exon, introduces a stop codon $\left(\mathrm{Q} 455^{\star}\right)$ just before the second predicted transmembrane domain. A second allele, $i a v^{3621}$, was isolated by screening chemically mutagenized chromosomes for slow recovery after $\mathrm{CO}_{2}$ anesthesia, another phenotype of $i a v^{1}$ (data not shown). This mutation, which shows a milder locomotor phenotype than $i a v^{1}$ in hemizygous males (see Materials and Methods), deletes 12 base pairs, encoding amino acids 388-391 ( $\triangle$ TFAQ), from exon 3 . The deletion, located just before the first transmembrane domain, includes a position at which an aromatic amino acid (F/Y) is conserved in all TRPV proteins.

\section{A hearing defect in iav mutants}

The only other Drosophila TRPV channel subunit, which is encoded by the nanchung locus, is expressed in chordotonal neurons and is required for sound transduction by the antennal chordotonal organ (Kim et al., 2003). We measured the response of iav mutants and controls to near-field sound by recording extracellular potentials from the antenna while the distal antennal segments were vibrated by near-field sound stimuli; this signal reflects the aggregate response of the neurons in Johnston's organ (Eberl et al., 2000). Sound pulses evoked clearly correlated potential changes in 40 of 43 antennae on control flies (Fig. 2). In contrast, antennal sound-evoked potentials were completely absent in $i a v^{1} / Y$ males (15 antennae, 8 flies), $\operatorname{iav}^{3621} / Y$ males (12 antennae, 6 flies), and $i a v^{1} / i a v^{3621}$ females ( 10 antennae in 5 flies) (data not shown), even in response to high-amplitude stimulation or when responses to 100 pulse trains are averaged (Fig. 2). A $9.2 \mathrm{kbp}$ segment of genomic DNA including the complete CG4536 gene (Fig. 1C) (see Materials and Methods) restored sound-evoked potentials and full locomotor activity to iav mutants in three of four transgenic lines (Fig. 2).

\section{The IAV protein mediates hypotonically induced $\mathrm{Ca}^{2+}$ increases and cation currents}

Expressing the NAN protein in cultured cells promoted inward currents and intracellular calcium increases in response to hypoosmotic perfusion (Kim et al., 2003). We examined whether the IAV channel possesses similar properties. CHO-K1 cells were transfected with iav cDNA and loaded with the calcium indicator Fluo-4/AM. Transfected cells, but not mock-transfected controls, showed spikes in intracellular calcium concentration after superfusion with a hypo-osmotic solution (Fig. 3A). The amplitude of the calcium spikes increased with decreasing osmolality (Fig. 3B). Addition of $10 \mathrm{~mm}$ EGTA to the hypo-osmotic medium abolished the response (data not shown), indicating that an influx of extracellular $\mathrm{Ca}^{2+}$ is required for the response. IAV-expressing cells were unresponsive to stimuli that can activate other TRP family members, including capsaicin, menthol, and temperatures ranging from 10 to $60^{\circ} \mathrm{C}$ (data not shown).

To determine whether the IAV protein mediates $\mathrm{K}^{+}$and $\mathrm{Na}^{+}$ currents, we recorded currents from HEK293T cells expressing iav cDNA in whole-cell patch configuration (Fig. 3C,D). Enhanced GFP (EGFP) cDNA was cotransfected with iav cDNA, and cells transfected with EGFP alone were used as a negative control. Ionic currents were recorded in response to a ramped voltage command ranging from -100 to $100 \mathrm{mV}$ in the absence or presence of hypotonic solution. The slope conductance of cells expressing the IAV channel, measured 5 min after exchange of bath solution to hypotonic solution, was $0.73 \pm 0.13 \mathrm{nS}(n=5)$,
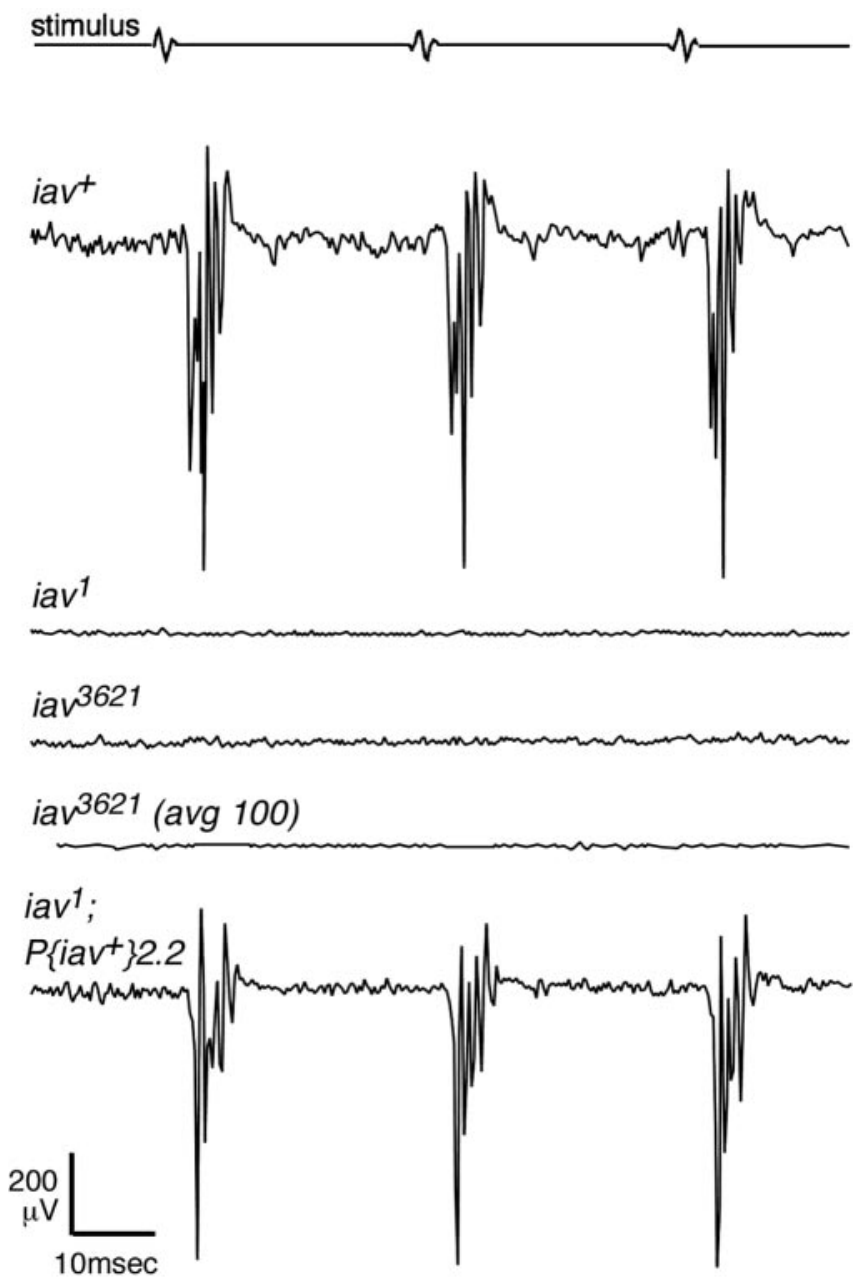

Figure 2. A hearing defect in iav mutants. Recordings of antennal sound-evoked potentials from control $\left(i a{ }^{+}\right)$, iav mutant, and transgenic iav; $P\left[i a v^{+}\right]$flies in response to repeated pulse trains. Records shown are averaged responses in single antennae to 10 or (where noted) 100 pulse stimuli. Mutants hemizygous for either iav allele show no trace of a response, whereas a single wild-type transgene $P\left[\mathrm{iav}^{+}{ }^{+}\right.$2.2] restores a normal response to the iav $^{7}$ background.

whereas the slope conductance of cells expressing only EGFP was $0.39 \pm 0.06 \mathrm{nS}(n=5)$. The basal level of osmo-sensitive conductance in control cells may be attributable to expression of endogenous osmo-sensitive channels by the HEK293T cells, which are derived from kidney (Liedtke et al., 2000). Thus, expression of the IAV channel resulted in a $0.34 \mathrm{nS}$ increase in hypotonically induced conductance. The reversal potential of ionic currents through the IAV channel is $\sim 0 \mathrm{mV}$, and the ratios of $\mathrm{K}^{+}$to $\mathrm{Na}^{+}$ permeability $\left(\mathrm{P}_{\mathrm{K}} / \mathrm{P}_{\mathrm{Na}}\right)$ and $\mathrm{Ca}^{2+}$ to $\mathrm{Na}^{+}\left(\mathrm{P}_{\mathrm{Ca}} / \mathrm{P}_{\mathrm{Na}}\right)$ were $0.85 \pm$ $0.01(n=3)$ and $2.79 \pm 0.03(n=4)$, respectively. These results indicate that IAV expression can promote the formation of a nonselective cation channel that is activated by hypotonic stress.

\section{IAV expression and localization to cilia}

In situ hybridization with iav antisense probes to Drosophila embryos revealed iav transcripts in chordotonal neurons of the embryonic PNS (Fig. 4A), with no detectable expression elsewhere. To visualize the IAV protein, we generated two antisera against its cytoplasmic $\mathrm{N}$ - and C-terminal regions. Both antisera detected a band at $\sim 121 \mathrm{kDa}$, close to the expected size ( $124 \mathrm{kDa})$ of the IAV protein, on Western blots of membrane extracts from HEK293 cells expressing IAV but not control cell extracts (data not 
A

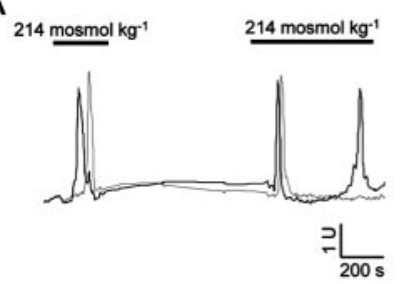

C

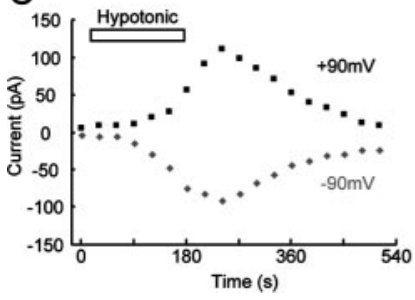

B
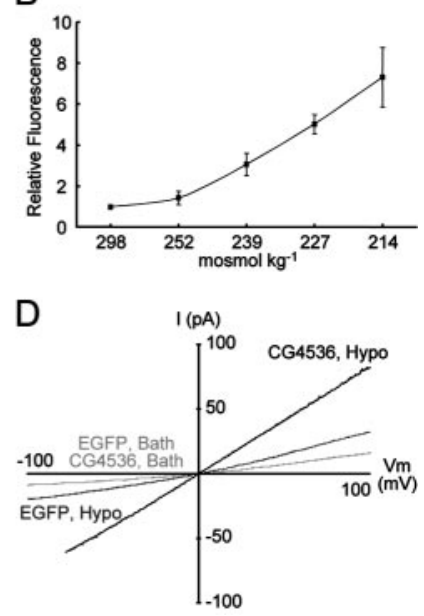

Figure 3. The IAV channel is gated in response to hypo-osmotic stress. A, IAV-expressing cells exhibit single $\mathrm{Ca}^{2+}$ peaks (gray) or oscillations (black) during prolonged hypo-osmotic stimulation $\left(214 \mathrm{~m} 0 \mathrm{sm} / \mathrm{kg}, 2 \mathrm{~mm} \mathrm{Ca}{ }^{2+}\right)$. U, Arbitrary unit of $\mathrm{Ca}^{2+}$ signals quantified by confocal microscope. $B$, Dependence of $\mathrm{Ca}^{2+}$ spike amplitude on osmolality. The mean amplitudes of the $\mathrm{Ca}^{2+}$ peak in response to hypotonic solutions $(214,227,239,252 \mathrm{m0sm} / \mathrm{kg})$ are normalized by the $\mathrm{Ca}^{2+}$ signals in isotonic solution $(298 \mathrm{~m} 0 \mathrm{sm} / \mathrm{kg})$. Error bars represent SEM. Data were recorded from $>100$ cells in five independent experiments. C, D, lonic currents and $I-V$ relationship stimulated by hypotonic solutions $(221 \mathrm{~m} 0 \mathrm{sm} / \mathrm{kg})$ in IAV-expressing cells in a whole-cell patch configuration. Step and ramp voltage clamp was applied to IAV channeltransfected HEK293T cells, and the resulting currents were recorded. IAV channel showed delayed activation after hypotonic solution treatment, and the reversal potential was $\sim 0 \mathrm{mV}$.

shown). Staining cryostat sections of legs, wings (data not shown), and antennae with either antiserum, together with the neural-specific cytoskeletal label mAb $22 \mathrm{C} 10$, showed that IAV is expressed specifically in chordotonal neurons and is localized to their ciliary outer segments (Fig. $4 B$ ). The IAV immunoreactivity is absent in $i a v^{1}$ and $i a v^{3621}$ mutant flies (Fig. 4C), indicating that these alleles are both protein nulls.

Because IAV is expressed in the same cells and localized to the same site as NAN (Kim et al., 2003), we asked whether IAV localization in sensory cilia depends on the presence of NAN. Immunostaining of nan mutant antennae with anti-IAV sera showed no detectable IAV in the cilia (Fig. 4D). Conversely, immunostaining of $i a v^{1}$ mutant antennal sections with anti-NAN serum revealed no detectable NAN in the cilia (Fig. $4 F$ ). Thus, IAV and NAN are mutually dependent for their stability and/or localization to the cilium.

\section{IAV is restricted to the proximal part of the cilium}

To label IAV in vivo, we inserted the EGFP coding sequence into a genomic rescue construct in either the $\mathrm{N}$ - or C-terminal cytoplasmic regions of IAV (supplemental material, available at www.jneurosci.org). $i a v^{l}$ mutants expressing the N-terminal GFP insertion construct showed little or no rescue of sound-evoked potentials, and no GFP signal was detected in the cilia or neurons. However, the C-terminal GFP insertion completely rescued the mutant electrophysiology, indicating that this fusion protein was expressed and fully functional in chordotonal organs (data not shown). Consistent with the anti-IAV labeling, GFP fluorescence was observed in the chordotonal cilia of Johnston's organ (Fig. $5 A-C$ ) and most clearly in larval chordotonal organs (Fig. 6A-C). As for endogenous IAV, the localization of IAV-GFP was dependent on NAN; no ciliary GFP signal was detected in nan mutants containing the same IAV-GFP construct that localized to cilia in wild type (Fig. 5D-F).
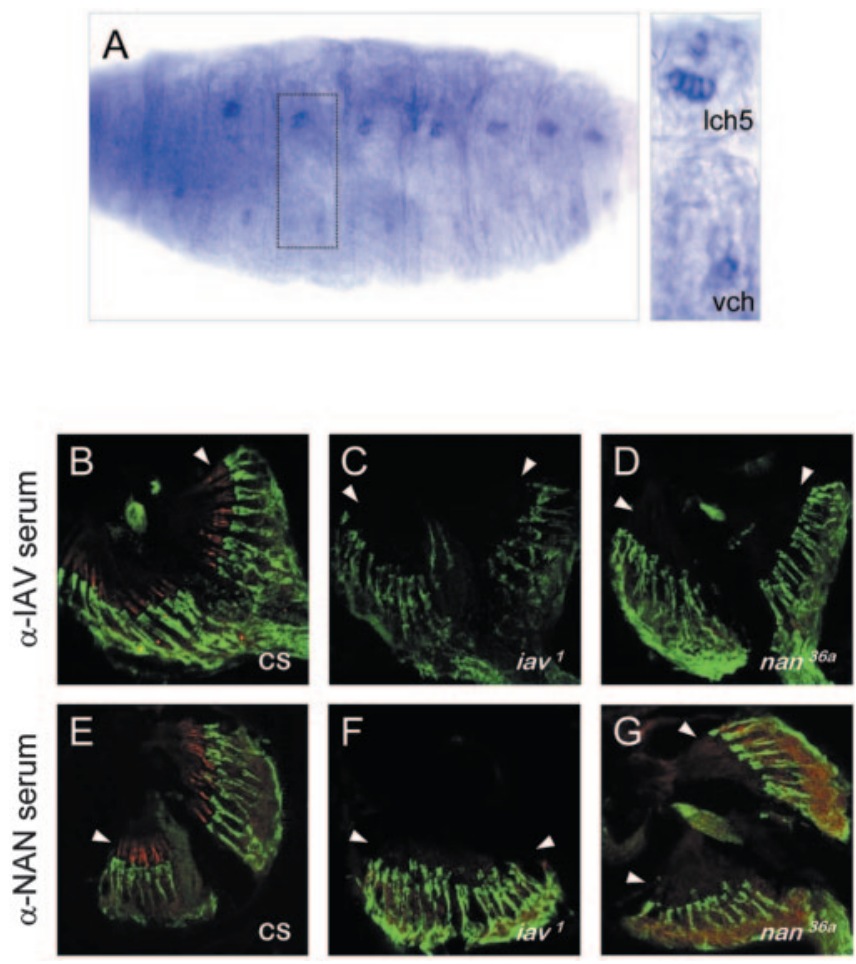

Figure 4. Expression, localization, and interdependence of IAV and NAN in chordotonal cilia. $A$, In situ hybridization of an iav antisense RNA probe to embryos at late stage 16. Lateral (Ich) and ventral (vch) chordotonal organs are indicated and enlarged in the inset. $B-G$, Immunostaining of the second antennal segment of wild-type $(B, E), \operatorname{iav}^{1}(C, F)$, and $\operatorname{nan}^{36 a}(D, G)$ with anti-IAV or anti-NAN serum (red). The anti-IAV serum was directed against the $\mathrm{N}$-terminal cytoplasmic region (anti-IAV-N; see Materials and Methods); similar results were seen with an anti-IAV C-terminal antiserum (data not shown). The mAb 22C10 (Zipursky et al., 1984), which labels a neural cytoskeletal protein in the cell bodies and inner segments but not the ciliary outer segments, is shown in green. The arrowheads denote the position of the outer segments.

Each chordotonal cilium has a conventional $9+0$ axonemal cytoskeleton, but this is interrupted at approximately two-thirds of its length by a ciliary dilation, where the ciliary membrane and axonemal microtubule pairs bulge outwards to enclose a tubular array of unknown composition and function. The dendritic cap, the extracellular matrix to which the cilium is attached, contacts the cilium only distal to the dilation (Fig. 6D). In embryonic chordotonal neurons, IAV-GFP extends up to but not beyond the ciliary dilation (Fig. $6 B, C, E$ ), and thus is absent from the zone of contact with the dendritic cap.

\section{Discussion}

Previously, we showed that the Drosophila TRPV channel NAN is required for hearing (Kim et al., 2003). Here, we show that the only other Drosophila TRPV channel protein is encoded by the iav gene. Like NAN, IAV is gated by hypotonic stress in vitro; the endogenous protein is specifically expressed in chordotonal neurons and localized to their cilia and is required for auditory transduction. Interestingly, localization of either NAN or IAV in the cilia is in each case dependent on the presence of the other protein, indicating that NAN-IAV interactions are required for channel stability and/or localization. Finally, we show that IAV is restricted to the proximal part of the cilia, suggesting that activating forces are transmitted to the IAV-NAN channels via the ciliary membrane or cytoskeleton.

The interdependence of IAV and NAN is consistent with the complete absence of transduction in nan and iav mutants but 
contrasts with the ability of each protein to promote a hypotonically activated current when individually expressed in cell culture. This suggests that either endogenous TRPV subunits in the cultured cells can heteromultimerize with the expressed TRPV channels, or that homomultimeric channels are more stable in cultured cells than in chordotonal neurons. These lowabundance channels may only be detectable if concentrated in the cilium; homomultimers may not be visible if they are excluded from the cilium.

These data are comparable with the functional interdependence of OSM-9 and OCR channel proteins in Caenorhabditis (Tobin et al., 2002). OSM-9, the nematode protein most similar to IAV, is expressed in diverse sensory neurons, interneurons, and rectal gland cells, whereas the four different OCR proteins are expressed in more restricted subsets of sensory neurons or in rectal gland cells. Coexpression of OSM-9 and OCR-2 in the ciliated ASH neuron is required for their localization to the cilium and for responses to aversive odorants, hyperosmotic stimuli, and touch (Tobin et al., 2002). OCR-4, the nematode protein most similar to NAN, is expressed together with OSM-9 only in the mechanosensitive OLQ neurons. These cells, which, like the Drosophila chordotonal neurons, have a differentiated cilium and extended ciliary rootlet, could be the nematode version of chordotonal organs.

\section{Models for channel activation}

How is the NAN/IAV channel gated? TRPV channels can be activated by diverse physical factors including temperature and hypotonic stress (Nilius et al., 2004), whereas OSM-9, the founding member of the family, was first identified by its requirement in the transduction of hyperosmotic stimuli and nose touch. However, the structural basis for TRPV channel gating is not yet known. Several TRPVs open in response to hypotonic stress or cell swelling, but this is not necessarily because of direct gating by membrane tension; TRPV4 channels in cell-attached patches could not be opened by applied pressure (Strotmann et al., 2000). Divergent evidence implicates either phosphorylation or arachidonic acid signaling in the indirect gating of mammalian TRPV4 by hypo-osmotic stimuli (Xu et al., 2003; Nilius et al., 2004), but neither pathway is fast enough to account for the speed of acoustic transduction. The Drosophila Johnston's organ can transduce signals up to $500 \mathrm{~Hz}$ with millisecond latencies (Eberl et al., 2000).

A prevailing conceptual model for direct mechanogating (Ernstrom and Chalfie, 2002; Sukharev and Corey, 2004), based on studies of vertebrate hair cells and C. elegans mutants defective in body touch, posits a transcellular complex in which the gated channel is anchored to both the cytoskeleton and to an extracellular link or matrix; relative movement of the intracellular and extracellular structures opens the channel. These elements are indeed present in chordotonal organs. The ciliary axoneme provides an extended cytoskeleton to which the TRPV channels could be anchored, whereas a specially shaped extracellular matrix, the dendritic cap, attaches to the distal tip of the cilium. In mutants lacking NOMPA, a ZP-domain cap protein, the cap is disorganized and detached from the cilia, and transduction is
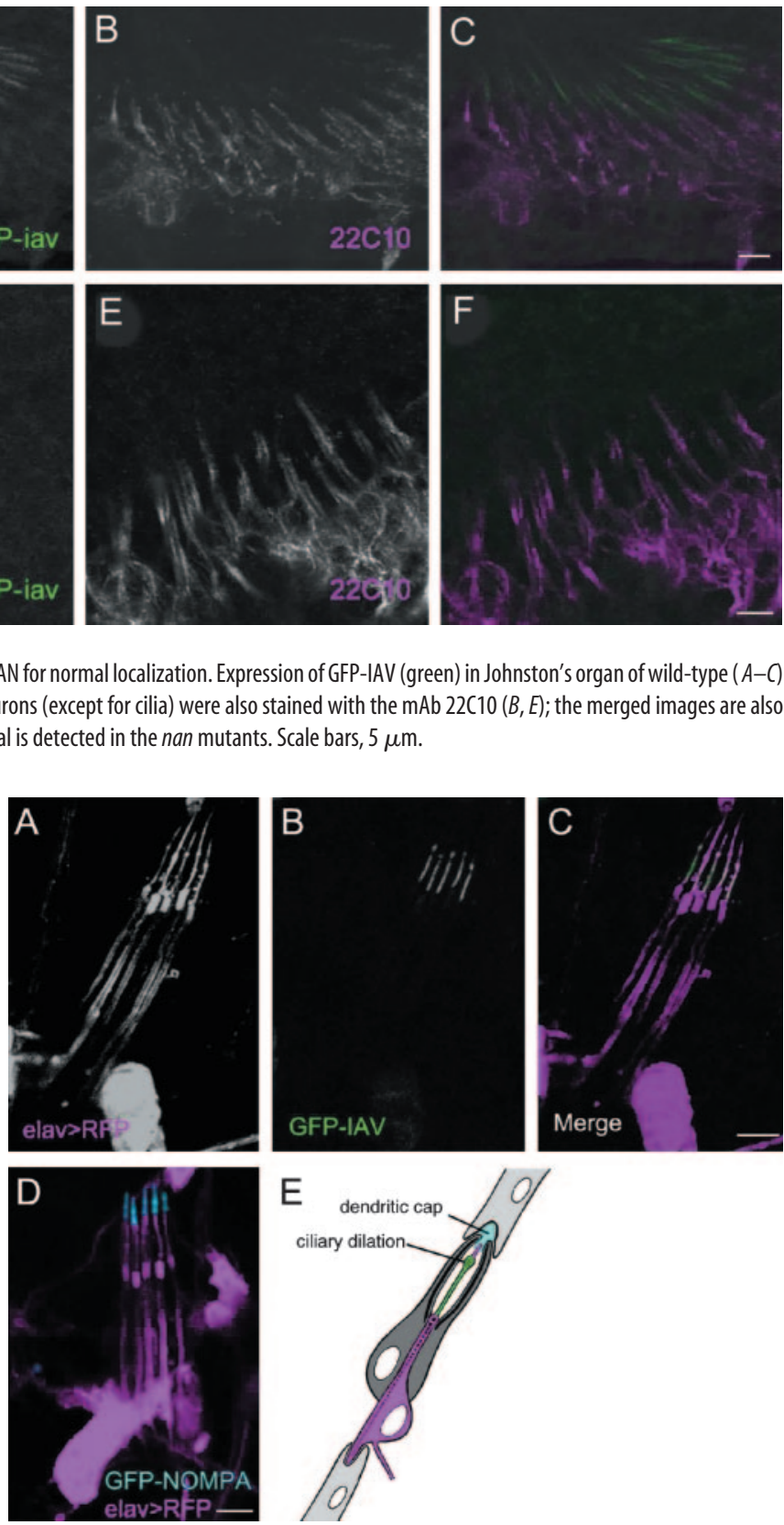

GFP-iav
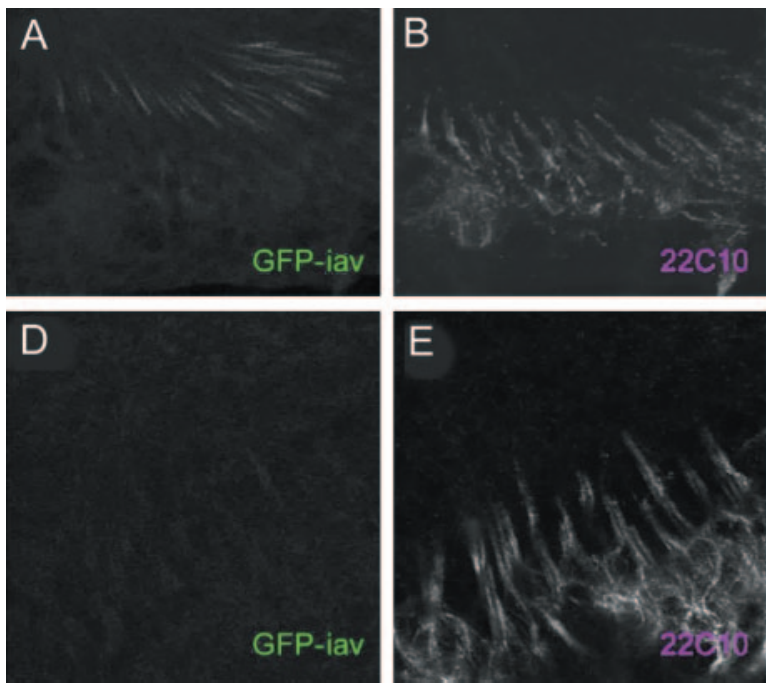

Figure 5. GFP-IAV requires NAN for normal localization. Expression of GFP-IAV (green) in Johnston's organ of wild-type ( $A-C)$ and nan mutant $(D-F)$ flies. Neurons (except for cilia) were also stained with the $\mathrm{mAb} 22 \mathrm{C} 10(B, E)$; the merged images are also shown $(C, F)$. No ciliary GFP signal is detected in the nan mutants. Scale bars, $5 \mu \mathrm{m}$.

Figure 6. Restricted localization of IAV in chordotonal cilia. Expression of a functional GFPIAV (green) or GFP-NOMPA (cyan) transgene in larval chordotonal organs (Ich5) showing proximal versus distal intracellular localization. Neurons are counter labeled with RFP (magenta) expressed under the control of the neural-specific elav-GAL4 driver. $A$, Elav-driven RFP; $B$, GFPIAV; $C$, merge of $A$ and $B ; D$, GFP-NOMPA (cyan) and RFP; $E$, schematic of a single larval scolopidium (Chung et al., 2001) showing the position of the ciliary dilation and dendritic cap. GFP-IAV labeling is restricted to the region proximal to the ciliary dilation, whereas NOMPA-RFP is distal to the dilation. Scale bars, $5 \mu \mathrm{m}$.

eliminated (Chung et al., 2001). However, the restricted localization of IAV in the proximal part of the cilium means that the IAV-NAN channel cannot interact directly with cap components. If NAN and IAV are mechanically gated, the forces that gate the IAV/NAN channel may be transmitted down the axoneme through the ciliary membrane or via other extracellular material in the scolopale space that encloses the cilium.

The involvement in transduction of a more distally located channel cannot be excluded. One candidate is NOMPC, the TRPN channel that mediates the mechanoreceptor current in 
mechanosensory bristles (Walker et al., 2000). Sound-evoked potentials, which reflect the aggregate activity of many individual chordotonal neurons, are reduced by approximately half in nompC null mutants (Eberl et al., 2000), suggesting either an absolute requirement for NOMPC in some chordotonal neurons or a partial contribution to transduction in all of them. In contrast to the limited area of cap/cilium contact in chordotonal organs, the entire ciliary outer segment in a bristle neuron is ensheathed by the dendritic cap. Although NOMPC has never been definitively localized, its modest contribution to chordotonal transduction could reflect a NOMPC-cap interaction restricted to the distal cilium. Because nompC null mutants retain substantial sound-evoked potentials but iav and nan mutants eliminate them completely, NOMPC activity, even if present, is not required to activate the IAV/NAN channels. Conversely, the TRPV channels are not expressed in bristles or other external sensory organs, and thus are not the source of the residual, nonadapting, bristle mechanoreceptor current in nompC mutants.

\section{Other inactive phenotypes}

Previously described phenotypes of $i a v^{1}$ mutants include locomotor inactivity, courtship abnormalities, and altered responses to cocaine (Kaplan, 1977; O’Dell et al., 1987; O’Dell, 1993, 1994; McClung and Hirsh, 1999). In addition, reduced levels of octopamine and tyramine have been reported in iav (O'Dell et al., 1987; McClung and Hirsh, 1999), but we have not been able to repeat these observations using more advanced analytical methods (S. Cole and J. Hirsh, unpublished observation). Rearing iav mutants on tyramine-supplemented medium did not restore sound-evoked potentials (data not shown).

Does the defect in chordotonal transduction underlie the full range of phenotypes seen in iav mutants? Both nan (Kim et al., 2003) and other deaf mutants (Eberl et al., 2000) also show sedentary behavior, as does the $i a v^{3621}$ allele described here, and similarly, antennal amputation also causes sedentary behavior (data not shown). These observations imply that chordotonal sensory input is needed for normal levels of locomotor activity. However, $i a v^{1}$ mutants are less active than either nan or $i a v^{3621}$ mutants, although all three mutants completely lack soundevoked potentials. This suggests that another IAV function, undetectable by auditory recording, is retained in the $i a v^{3621}$ and nan mutants but not in $i a v^{1}$ perhaps at an extraciliary site in chordotonal neurons or elsewhere in the nervous system. Precedent for a nontransducing role for IAV may be found in the OCR-independent expression of OSM-9 in C. elegans AWC neurons, where OSM-9 is located in the cell body and required for olfactory adaptation (Tobin et al., 2002). We can rule out the possibility that the $i a v^{1}$ mutant chromosomes carry a second-site mutation that contributes independently to inactivity, because this phenotype is fully rescued by iav transgenes, but a linked enhancer of the iav phenotype remains a possibility. Targeting expression of $i \mathrm{av}^{+}$specifically to chordotonal organs and additional testing of neurotransmitter levels and drug interactions in $i a v^{3621}$, nan, and other deaf mutants and transgenic animals may help to clarify this issue.

The alterations in cocaine responses observed in avv $^{1}$ (McClung and Hirsh, 1999) and $i a v^{3621}$ (data not shown) cannot yet be interpreted in terms of TRPV channel activity, because the $i a v^{+}$transgene insertions that restore evoked auditory potentials and locomotor activity do not appear to rescue the cocaine response phenotypes seen in $i a v^{1}$ or $i a v^{3621}$ (Z. Gong, J. Young, and Hirsh, unpublished observations). A further indication that the cocaine responses are independent of auditory transduction comes from the observation that nan flies show normal cocaine responses as do flies made deaf by amputation of their antennae (Gong and Hirsh, unpublished observations). Our favored interpretation of these findings is that there is a secondary site of low level but functionally important IAV expression within the nervous system that is not restored by the regulatory elements included in the $\mathrm{iav}^{+}$transgenes used in this study. In any case, these observations point to the possibility of a divergent role for the IAV TRPV channel that may be independent of NAN and independent of the role of IAV in auditory transduction.

\section{References}

Benham CD, Gunthorpe MJ, Davis JB (2003) TRPV channels as temperature sensors. Cell Calcium 33:479-487.

Caterina MJ, Julius D (2001) The vanilloid receptor: a molecular gateway to the pain pathway. Annu Rev Neurosci 24:487-517.

Caterina MJ, Schumacher MA, Tominaga M, Rosen TA, Levine JD, Julius D (1997) The capsaicin receptor: a heat-activated ion channel in the pain pathway. Nature 389:816-824

Caterina MJ, Rosen TA, Tominaga M, Brake AJ, Julius D (1999) A capsaicin-receptor homologue with a high threshold for noxious heat. Nature 398:436-441.

Caterina MJ, Leffler A, Malmberg AB, Martin WJ, Trafton J, Petersen-Zeitz KR, Koltzenburg M, Basbaum AI, Julius D (2000) Impaired nociception and pain sensation in mice lacking the capsaicin receptor. Science 288:306-313.

Chung Y, Zhu J, Han Y, Kernan M (2001) nompA encodes a PNS-specific, ZP-domain protein required to connect mechanosensory dendrites to sensory structures. Neuron 29:415-428.

Colbert H, Smith T, Bargmann C (1997) OSM-9, a novel protein with structural similarity to channels, is required for olfaction, mechanosensation, and olfactory adaptation in Caenorhabditis elegans. J Neurosci 17:8259-8369.

Corey DP (2003) New TRP channels in hearing and mechanosensation. Neuron 39:585-588.

Curtin KD, Zhang Z, Wyman RJ (1999) Drosophila has several genes for gap junction proteins. Gene 232:191-201.

Eberl DF (1999) Feeling the vibes: chordotonal mechanisms in insect hearing. Curr Opin Neurobiol 9:389-393.

Eberl DF, Duyk GM, Perrimon N (1997) A genetic screen for mutations that disrupt an auditory response in Drosophila melanogaster. Proc Natl Acad Sci USA 94:14837-14842.

Eberl DF, Hardy RW, Kernan MJ (2000) Genetically similar transduction mechanisms for touch and hearing in Drosophila. J Neurosci 20:5981-5988.

Ernstrom GG, Chalfie M (2002) Genetics of sensory mechanotransduction. Annu Rev Genet 36:411-453.

Gao X, Wu L, O'Neil RG (2003) Temperature-modulated diversity of TRPV4 channel gating: activation by physical stresses and phorbol ester derivatives through protein kinase C-dependent and -independent pathways. J Biol Chem 278:27129-27137.

Gillespie PG, Walker RG (2001) Molecular basis of mechanosensory transduction. Nature 413:194-202.

Hall JC (1992) iav. In: The genome of Drosophila melanogaster (Lindsley LL, Zimm GG, eds), p 285. San Diego: Academic.

Homyk T, Sheppard DE (1977) Behavioral mutants of Drosophila melanogaster. I. Isolation and mapping of mutations which decrease flight ability. Genetics 87:95-104.

Kaplan WD (1977) iav: inactive. Drosophila Info Serv 52:1.

Kim J, Chung YD, Park DY, Choi S, Shin DW, Soh H, Lee HW, Son W, Yim J, Park CS, Kernan MJ, Kim C (2003) A TRPV family ion channel required for hearing in Drosophila. Nature 424:81-84.

Lewis EB, Bacher F (1968) EMS mutagenesis of Drosophila. Drosophila Info Serv 43:193.

Liedtke W, Friedman JM (2003) Abnormal osmotic regulation in trpv4-/mice. Proc Natl Acad Sci USA 100:13698-13703.

Liedtke W, Choe Y, Marti-Renom MA, Bell AM, Denis CS, Sali A, Hudspeth AJ, Friedman JM, Heller S (2000) Vanilloid receptor-related osmotically activated channel (VR-OAC), a candidate vertebrate osmoreceptor. Cell 103:525-535.

McClung C, Hirsh J (1999) The trace amine tyramine is essential for sensitization to cocaine in Drosophila. Curr Biol 9:853-860. 
Nilius B, Vriens J, Prenen J, Droogmans G, Voets T (2004) TRPV4 calcium entry channel: a paradigm for gating diversity. Am J Physiol Cell Physiol 286:C195-C205.

O’Dell K, Coulon J-F, David J-C, Papin C, Fuzeau-Braesch S, Jallon J-M (1987) La mutation inactive produit une diminution marquee d'octopamine dans le cerveau des Drosophiles. CR Acad Sci Paris T. 305:199-202.

O'Dell KM (1993) The effect of the inactive mutation on longevity, sex, rhythm and resistance to p-cresol in Drosophila melanogaster. Heredity 70:393-399.

O'Dell KM (1994) The inactive mutation leads to abnormal experiencedependent courtship modification in male Drosophila melanogaster. Behav Genet 24:381-388.

Preston CR, Engels WR (1996) P-element-induced male recombination and gene conversion in Drosophila. Genetics 144:1611-1622.

Rehm EJ (2003) Inverse PCR and cycle sequencing of P element insertions for STS generation. In: Berkeley Drosophila genome project. Available at www.fruitfly.org/p_disrupt/inverse_pcr.html.

Sidi S, Friedrich RW, Nicolson T (2003) NompC TRP channel required for vertebrate sensory hair cell mechanotransduction. Science 301:96-99.

Skeath JB, Carroll SB (1994) The achaete-scute complex: generation of cellular pattern and fate within the Drosophila nervous system. FASEB J 8:714-721.

Spradling AC, Rubin GM (1982) Transposition of cloned P elements into Drosophila germ line chromosomes. Science 218:341-347.

Strassmaier M, Gillespie PG (2002) The hair cell's transduction channel. Curr Opin Neurobiol 12:380-386.

Strotmann R, Harteneck C, Nunnenmacher K, Schultz G, Plant TD (2000)
OTRPC4, a nonselective cation channel that confers sensitivity to extracellular osmolarity. Nat Cell Biol 2:695-702.

Sukharev S, Corey DP (2004) Mechanosensitive channels: multiplicity of families and gating paradigms. Sci STKE 2004:re4.

The FlyBase Consortium (2003) The FlyBase database of the Drosophila genome projects and community literature. Nucleic Acids Res 31:172-175.

Tobin D, Madsen D, Kahn-Kirby A, Peckol E, Moulder G, Barstead R, Maricq A, Bargmann C (2002) Combinatorial expression of TRPV channel proteins defines their sensory functions and subcellular localization in $C$. elegans neurons. Neuron 35:307-318.

Vriens J, Watanabe H, Janssens A, Droogmans G, Voets T, Nilius B (2004) Cell swelling, heat, and chemical agonists use distinct pathways for the activation of the cation channel TRPV4. Proc Natl Acad Sci USA 101:396-401.

Walker RG, Willingham AT, Zuker CS (2000) A Drosophila mechanosensory transduction channel. Science 287:2229-2234.

Wolff T (2000) Histological techniques for the Drosophila eye part II: adult In: Drosophila protocols (Sullivan W, Ashburner M, Hawley RS, eds), pp 229-234. Cold Spring Harbor, New York: Cold Spring Harbor Laboratory.

Xu H, Zhao H, Tian W, Yoshida K, Roullet JB, Cohen DM (2003) Regulation of a TRP channel by tyrosine phosphorylation: Src family kinasedependent phosphorylation of TRPV4 on Y253 mediates its response to hypotonic stress. J Biol Chem 278:11520-11527.

Zipursky SL, Venkatesh TR, Teplow DB, Benzer S (1984) Neuronal development in the Drosophila retina: monoclonal antibodies as molecular probes. Cell 36:15-26. 